\title{
USO DEL CINE COMERCIAL EN LA ENSEÑANZA DE PSICOLOGÍA PARA ESTUDIANTES DE MEDICINA Y DE BIOLOGÍA
}

\section{Use of commercial movies in Psychology teaching for Medicine and Biology students}

Jorge PÉREZ'; Eva BAILLÈS²

${ }^{1}$ Grupo de Investigación Educativa en Ciencias de la Salud (GRECS). Universidad Pompeu Fabra. Barcelona (España). ${ }^{2}$ Hospital Nostra Senyora de Meritxell. Andorra la Vella (Andorra).

Correo electrónico: jordi.perez@upf.edu

Fecha de recepción: 9 de junio de 2020

Fecha de aceptación: 19 de junio de 2020

Fecha de publicación: 15 de diciembre de 2020

\section{Resumen}

En este escrito se narra una experiencia de uso de tres películas comerciales para llevar a cabo evaluaciones formativas en Psicología con estudiantes de Medicina y de Biología. En ambos casos, los alumnos visionaban las películas en horas de clase y posteriormente realizaban el examen formativo. Unos días después, se entregaban a los estudiantes los exámenes corregidos para realizar una sesión de retroalimentación. En ésta se comentaban todas las preguntas y se hacía hincapié en los conceptos que no habían sido asimilados de forma correcta. Se hace una valoración muy positiva de la experiencia tanto por la alta satisfacción mostrada por los estudiantes como por el éxito académico final de éstos.

Palabras clave: «cinemeducation»; evaluaciones formativas; estudiantes de medicina; estudiantes de biología.

\section{Abstract}

In the present paper, we explain our experience using three popular/commercial films to carry out formative assessment in teaching Psychology to students of Medicine and Biology. In both cases, the students watched the films during the intended class hours, and the, following day they completed a formative exam. A few days later, the corrected exams were given back to the students in order to carry out a feedback session. The aim was to deal with all their questions 


\section{USO DEL CINE COMERCIAL EN LA ENSEÑANZA DE PSICOLOGÍA PARA ESTUDIANTES DE MEDICINA Y DE BIOLOGÍA JORGE PÉREZ; EVA BAILLÈS}

but focussing more closely on those answered with a lesser degree of success. We make a very positive evaluation of our experience, not only because of the satisfaction of the students but also because of their academic performance.

Key words: cinemeducation; formative assessment; medical students; biological students.

\section{Introducción}

El uso de películas populares con fines docentes ha sido y sigue siendo utilizado en estudios universitarios muy diversos tanto en ciencias sociales como en ciencias de la salud. Entre otras, tenemos constancia de experiencias en la enseñanza del derecho ${ }^{1}$, de la economía ${ }^{2}$, de la administración educativa ${ }^{3}$, de la rehabilitación ${ }^{4}$, de la farmacia ${ }^{5}$ o de la biología ${ }^{6-8}$; pero la utilización del cine comercial ha sido especialmente relevante en la enseñanza de la medicina ${ }^{9}$ y de la psicología ${ }^{10}$.

Además del uso del cine comercial en la enseñanza de la psiquiatría en los estudios de medicina ${ }^{11}$, en psicología se han realizado experiencias pedagógicas muy diversas en diferentes áreas, especialmente en psicopatología ${ }^{12}$. También conocemos experiencias educativas con el cine en cursos de introducción a la psicología ${ }^{13}$ y sobre diversas ramas de dicha ciencia como sería la psicología jurídica ${ }^{14,15}$, la del desarrollo ${ }^{16}$, la cognitiva ${ }^{17}$, la socia $\left.\right|^{18}$ o la industrial y organizacional ${ }^{19}$. Además los profesores de psicología tienen a su disposición diversas webs donde pueden encontrar listas de películas susceptibles de ser utilizadas en la enseñanza (https://www.mcgill. $\mathrm{ca} / \mathrm{tcpsych/teachingresources/films),} \mathrm{algunas}$ de ellas en español (https://www.lifeder.com/ peliculas-psicologicas).

Si bien el cine comercial ha sido frecuentemente usado en la enseñanza de la psicología con fines diversos, no conocemos experiencias donde haya sido usado con objetivos de evaluación de los aprendizajes. En el presente escrito pretendemos narrar nuestra experiencia de usar el cine como elemento clave para realizar evaluaciones formativas en la enseñanza de psicología en estudios de Medicina y de Biología.

\section{La experiencia con estudiantes de Medicina}

La actividad con estudiantes de medicina se realizó en la Facultad de Medicina de la Universidad Autónoma de Barcelona (UAB) durante los cursos 1995-96, 1996-97 y 1997-98. Los estudios de medicina de la UAB contemplaban dos asignaturas de psicología: «Bases psicológicas del comportamiento» y "Psicología Humana: bases sociales y conducta anormal». La primera materia era obligatoria e impartida por diversos profesores a todos los alumnos, repartidos en tres grupos de 90 estudiantes. La segunda era optativa y era desarrollada por un profesor diferente para cada uno de los tres grupos, de entre 30 y 75 estudiantes.

La experiencia que comentamos la realizamos en la enseñanza de la asignatura optativa «Psicología Humana: bases sociales y conducta anormal» que tenía 6 créditos y se impartía en el segundo semestre del segundo curso. Dado que previamente los estudiantes habían cursado la asignatura obligatoria, los objetivos docentes de nuestra materia se centraban en la psicología social y en la psicología anormal. Los temas tratados pueden verse en la tabla 1.

Durante el curso estaban previstas tres evaluaciones formativas relacionadas con la visualización de tres películas seleccionadas: El milagro de Anna Sullivan (1962) de Arthur Penn, Alguien voló sobre el nido del cuco (1975) de Milos Forman y La naranja mecánica (1971) de Stanley Kubrick.

\section{La experiencia con estudiantes de Biología}

La actividad con estudiantes de Biología se realizó en la Facultad de Ciencias de la Salud y de la Vida de la Universidad Pompeu Fabra (UPF) de Barcelona desde el curso 2001-02 hasta el 


\section{USO DEL CINE COMERCIAL EN LA ENSEÑANZA DE PSICOLOGÍA PARA ESTUDIANTES DE MEDICINA Y DE BIOLOGÍA JORGE PÉREZ; EVA BAILLÈS}

2006-07. Los estudios de Biología de la UPF se realizan desde 1998, con un número reducido de alumnos y un proyecto educativo innovador. La carrera tenía una orientación exclusivamente biosanitaria y se cursaba durante cinco años. Dicha licenciatura dio paso al actual grado de Biología Humana.

La experiencia que se narra se realizó en la asignatura "Psicología Humana» que tenía 4 créditos, era optativa y se impartía en el segundo trimestre del cuarto curso con un número de estudiantes que ha oscilado entre 27 y 42 . Los objetivos de la materia pretendían dar una visión general del comportamiento humano (procesos básicos y diferencias individuales), de las bases sociales de la conducta y del comportamiento anormal. Los temas previstos pueden verse en la tabla 1.

En dicha asignatura se realizaban dos evaluaciones formativas ligadas a dos de las películas citadas anteriormente: El milagro de Ana Sullivan y La naranja mecánica.

Tabla 1. Temas de las asignaturas susceptibles de evaluación en las diversas pruebas formativas

\begin{tabular}{|l|l|l|l|l|l|l|}
\hline \multicolumn{1}{|c|}{ Psicología Humana: Medicina UAB } & $\mathrm{PF}^{1}$ & $\mathrm{PF2}^{2}$ & PF3 $^{3}$ & \multicolumn{1}{|c|}{ Psicología Humana: Biología UPF } & PF1 $^{1}$ & PF2 $^{3}$ \\
\hline $\begin{array}{l}\text { 1. Procesos básicos y diferencias in- } \\
\text { dividuales }\end{array}$ & $\mathrm{X}$ & $\mathrm{X}$ & $\mathrm{X}$ & $\begin{array}{l}\text { 1. Percepción, memoria y pensa- } \\
\text { miento }\end{array}$ & $\mathrm{X}$ & $\mathrm{X}$ \\
\hline 2. Actitudes & $\mathrm{X}$ & $\mathrm{X}$ & $\mathrm{X}$ & 2. Aprendizaje & $\mathrm{X}$ & $\mathrm{X}$ \\
\hline 3. Interacción social & $\mathrm{X}$ & $\mathrm{X}$ & $\mathrm{X}$ & 4. Emoción & $\mathrm{X}$ & $\mathrm{X}$ \\
\hline 4. Agresión & $\mathrm{X}$ & $\mathrm{X}$ & $\mathrm{X}$ & 5. Inteligencia & $\mathrm{X}$ & $\mathrm{X}$ \\
\hline 5. Altruismo & $\mathrm{X}$ & $\mathrm{X}$ & $\mathrm{X}$ & 6. Personalidad & $\mathrm{X}$ & $\mathrm{X}$ \\
\hline 6. Atracción interpersonal & & $\mathrm{X}$ & $\mathrm{X}$ & $\begin{array}{l}\text { 7. Interacción social, actitudes } \\
\text { persuasión }\end{array}$ & $\mathrm{X}$ & $\mathrm{X}$ \\
\hline 7. Comportamiento sexual & & & $\mathrm{X}$ & 3. Motivación & $\mathrm{X}$ \\
\hline 8. Conducta anormal y tratamientos & & $\mathrm{X}$ & $\mathrm{X}$ & 8. Agresión & $\mathrm{X}$ & $\mathrm{X}$ \\
\hline 9. Conducta adictiva & & $\mathrm{X}$ & $\mathrm{X}$ & 9. Altruismo & $\mathrm{X}$ & $\mathrm{X}$ \\
\hline 10. Conducta antisocial & & & $\mathrm{X}$ & 10. Atracción interpersonal & & $\mathrm{X}$ \\
\hline 11. Estrés académico & & & & 11. Conducta sexual & $\begin{array}{l}\text { 12. Conducta anormal y tratamien- } \\
\text { tos }\end{array}$ & $\mathrm{X}$ \\
\hline 12. Personalidad y salud & & & $\mathrm{X}$ & 13. Conducta adictiva & 14. Conducta antisocial & $\mathrm{X}$ \\
\hline
\end{tabular}

$\mathrm{PF}=$ Prueba formativa; ${ }^{1}$ El milagro de Anna Sullivan; ${ }^{2}$ Alguien voló sobre el nido del cuco; ${ }^{3}$ La naranja mecánica

\section{Las películas seleccionadas}

Como hemos visto, a lo largo de los años hemos utilizado tres películas muy populares. En Medicina usamos las tres y en Biología dos ya que la asignatura tenía menos créditos. Las tres reúnen dos características claves para favorecer los aprendizajes de nuestros objetivos: por un lado son muy atractivas y amenas, y por otro sus argumentos se ajustan perfectamente a los temas tratados en las asignaturas.

Rev. Med. Cine. 2020; 16(4), 297-305 Ediciones Universidad de Salamanca / @®@ J. Med. Mov., 2020; 16(4), 297-305 


\section{USO DEL CINE COMERCIAL EN LA ENSEÑANZA DE PSICOLOGÍA PARA ESTUDIANTES DE MEDICINA Y DE BIOLOGÍA JORGE PÉREZ; EVA BAILLÈS}

a) El milagro de Anna Sullivan / The Miracle Worker (1962) de Arthur Penn está basada en hechos reales referentes a la vida de la escritora americana Helen Keller (1880-1968). Se centra en una joven Keller, ciega, sorda y muda desde la infancia, que es incontrolable y está a punto de ser enviada a una institución. Su incapacidad para comunicarse le genera frustración y conducta violenta. Sus padres buscan ayuda en un prestigioso centro que les proporciona una institutriz llamada Anna Sullivan, que también tiene problemas de visión, para encargarse de educar a su hija. A través de la persistencia, la terquedad y el cariño, Anna consigue que la niña llegue a comportarse correctamente y a comunicarse con sus seres queridos.

Esta película, ganadora de dos Óscar por los dos papeles femeninos, es ideal para ilustrar muchos procesos psicológicos, especialmente los relacionados con el aprendizaje (condicionamiento clásico, condicionamiento instrumental, reforzamiento positivo, reforzamiento negativo, castigo, etc.). Así mismo, permite mostrar la importancia de la adquisición del lenguaje en el comportamiento humano. Finalmente también es una joya para ilustrar los temas de la agresión, el altruismo y la atracción interpersonal.

b) La película Alguien voló sobre el nido del Cuco / One Flew Over the Cuckoo's Nest (1975) de Milos Forman, siempre tuvo una gran repercusión mediática al obtener cinco Óscar, entre ellos a la mejor película, al mejor director y a los mejores actores, masculino y femenino.

Narra la historia de McMurphy, un hombre con varias condenas, que se encuentra nuevamente en la cárcel acusado de violación. Convence a las autoridades de que está suficientemente loco para que lo envíen a un hospital psiquiátrico. Dentro de dicha institución simpatiza con diversos internos y genera diferentes problemas por no atenerse a las reglas del centro. Esto genera un duro y permanente enfrentamiento con la enfermera Ratched, importante responsable de la gestión de la vida hospitalaria.

A pesar de que la película ha sido criticada por dar una visión muy negativa hacia la psiquiatría, servía perfectamente para tratar la mayoría de nuestros objetivos docentes. Es especialmente útil para ilustrar los temas de liderazgo, del comportamiento grupal y de las terapias biológicas para modificar el comportamiento.

c) Finalmente, la película La naranja mecánica / A Clockwork Orange (1971) de Stanley Kubrick también es muy conocida por la repercusión que tuvo en su momento dado su argumento. Un joven adolescente británico llamado Alex dirige una banda de delincuentes juveniles que muestran una gran violencia. Detenido y encarcelado, se somete, para poder salir de la prisión, a un tratamiento conductual para reducir su comportamiento agresivo. La terapia consigue anular su agresividad pero queda indefenso al ser rechazado por su familia. El film está basado en la novela homónima de Anthony Burgess (1962), que es una crítica mordaz a la hipocresía de las principales instituciones políticas, sociales o religiosas.

Esta película ayudó a generar una actitud negativa hacía la terapia de conducta pero, a pesar de ello, pensamos que es excelente para ilustrar diversos temas de psicología social (comportamiento agresivo, liderazgo, tipo de crianza o comportamiento sexual) y el de las terapias del comportamiento.

Rev. Med. Cine. 2020; 16(4), 297-305 Ediciones Universidad de Salamanca / @@@ J. Med. Mov., 2020; 16(4), $297-305$ 


\section{USO DEL CINE COMERCIAL EN LA ENSEÑANZA DE PSICOLOGÍA PARA ESTUDIANTES DE MEDICINA Y DE BIOLOGÍA \\ JORGE PÉREZ; EVA BAILLÈS}

Tabla 2. Ejemplos de preguntas usadas en las evaluaciones formativas. Entre paréntesis y en negrita se hace referencia al tema del programa.

El milagro de Anna Sullivan

1. (Aprendizaje) En la película aparecen numerosas escenas relacionadas con el aprendizaje. En la secuencia de la lucha entre Helen (niña) y Anna (maestra) referente al aprendizaje de comer con cuchara en el plato aparecen dos procesos relevantes.

a) Cuando la niña estaba tumbada en el suelo dando patadas y Anna respondía con indiferencia, la maestra estaba intentando la conducta perturbadora de la niña.

b) Posteriormente cuando la niña realizaba conductas agresivas recibía respuestas aún más agresivas (golpes en la cara) por parte de Anna. La maestra estaba la conducta agresiva de la niña.

c) ¿En qué se parecen ambos procesos?

2. (Emociones) ¿Alguna escena te ha hecho recordar la universalidad de las expresiones faciales en las emociones?

3. (Pensamiento) Antes de la llegada de Anna, ¿̇crees que Helen podía desarrollar pensamiento? , ya que

\section{Alguien voló sobre el nido del cuco}

4. (Tratamientos) Indica los tipos de terapias biomédicas a las que fue sometido McMurphy durante su estancia en el Hospital.

5. (Conducta adictiva) Indica el tipo de drogas que son consumidas en la película (por cualquiera de los personajes) 6. (Diferencias individuales) Supongamos que la población masculina de la sociedad americana tiene una puntuación media en Susceptibilidad al Castigo (SC) de 11.0 (DT = 4.0) y en Susceptibilidad a la Recompensa (SR) de 12.0 (SD = 3.0).

Si encontraras un test anónimo con una puntuación directa de 19 en SC y de 5 en SR, ¿A quién pertenecería el resultado a McMurphy o a Billy? Razona la respuesta.

La naranja mecánica

7. (Motivación) La motivación de Alex en la prisión para colaborar con el cura de la prisión era (primaria o secundaria) y (intrínseca o extrínseca)

8. (Agresión) De las variables que fomentan la agresión, ¿Cuáles serían las más relevantes para explicar la primera sesión de ultraviolencia (agresión al escritor y su esposa)?

9. (Terapias psicológicas) El tratamiento Ludovico pretendía eliminar la conducta agresiva de Alex. De las técnicas cognitivo-conductuales que se utilizan para reducir comportamientos nocivos, ¿cuál es la que le aplicaron al protagonista?

\section{Las pruebas formativas}

Debido a su alto valor para el aprendizaje, las pruebas formativas eran obligatorias y consistían en unas 20 preguntas cortas o de complemento (poner respuestas concretas sobre espacios concretos) sobre aspectos psicológicos explicados en clase y relacionados con el argumento de la película. La tabla 2 muestra algunos ejemplos de preguntas concretas utilizadas en las evaluaciones. Las cuestiones de cada prueba se ajustaban al temario explicado hasta aquel momento, así en la última evaluación se podían hacer preguntas sobre todo el temario. En la tabla 1 puede verse los temas que se evaluaron en cada uno de los exámenes.

Al ser pruebas formativas el objetivo principal era favorecer el aprendizaje de los objetivos docentes previstos hasta el momento. Si el rendimiento del estudiante en el examen era negativo, no tenía ninguna consecuencia negativa para la nota final. Pero, para aumentar la motivación de

Rev. Med. Cine. 2020; 16(4), 297-305 Ediciones Universidad de Salamanca / @®@ J. Med. Mov., 2020; 16(4), 297-305 


\section{USO DEL CINE COMERCIAL EN LA ENSEÑANZA DE PSICOLOGÍA PARA ESTUDIANTES DE MEDICINA Y DE BIOLOGÍA JORGE PÉREZ; EVA BAILLÈS}

los estudiantes, éstos podían obtener un pequeño plus sobre la nota final si superaban el estándar habitual utilizado en nuestro país ( 5 sobre 10$)$. En general, los estudiantes podían obtener hasta 0,5 puntos sobre su nota final con las evaluaciones formativas. El plus era proporcional a la calificación obtenida en cada prueba siempre que se superara el mínimo requerido (5 sobre 10 ).

\section{Procedimiento}

Tanto en Medicina como en Biología, la metodología ha sido siempre la misma con la excepción de que en Medicina se usaron tres películas y en Biología dos. En primer lugar los estudiantes visionaban la película en el aula dentro del horario previsto en la asignatura. Al siguiente día de clase, los alumnos realizaban la prueba formativa que era corregida de forma inmediata por parte de los profesores. Durante la siguiente clase se repartían los exámenes corregidos y se realizaba una sesión de retroalimentación (feedback) comentando todas las preguntas, incidiendo en aquellas en las que las respuestas fueron menos exitosas. Al acabar esta clase de control se recogían los ejercicios que quedaban a disposición del profesor.

Las evaluaciones se realizaban a lo largo del curso manteniendo un espacio de tiempo semejante entre ellas. En Medicina se hacían tres, siendo la tercera al final. En Biología la primera se realizaba hacia la mitad del curso y la segunda al final. Para evitar el efecto halo (posibles sesgos), las correcciones de los exámenes se hacían siempre de forma anónima.

\section{Resultados académicos}

Para este trabajo presentamos un resumen de los resultados académicos en las pruebas formativas y las correlaciones encontradas entre dichas pruebas y las finales de Pruebas de Elección Múltiple (PEM). Las evaluaciones finales de las asignaturas comentadas además de las PEM también incluían pruebas de ensayo, pero hemos escogido el indicador de los resultados en las pruebas PEM por ser totalmente objetivas.

La tabla 3 presenta los rangos de porcentajes de alumnos que fracasaron (puntuaciones inferiores a 5) y que tuvieron gran éxito (notas de 70 superiores) en las pruebas. Como se puede observar, los resultados fueron muy positivos, donde una minoría de estudiantes, en algunos casos, no superó la puntuación mínima (entre el 0 y el 9\%). Por el contrario, el porcentaje de estudiantes con gran éxito fue muy elevado (entre el 36 y $75 \%$ en Medicina, y entre el 65 y el $100 \%$ en Biología).

Tabla 3. Rangos de porcentajes de estudiantes con fracaso (puntuaciones inferiores a 5) y con éxito destacado (puntuaciones de 7 o superiores).

\begin{tabular}{|c|c|c|c|}
\hline Prueba Formativa & Resultados & $\begin{array}{l}\text { Medicina UAB } \\
\text { (tres cursos) }\end{array}$ & $\begin{array}{l}\text { Biología UPF } \\
\text { (seis cursos) }\end{array}$ \\
\hline \multirow{2}{*}{ PF1 (El milagro de Anna Sullivan) } & Fracaso & $0-5 \%$ & $0-0 \%$ \\
\hline & Éxito notable & $43-63 \%$ & $79-100 \%$ \\
\hline \multirow{2}{*}{ PF2 (Alguien voló sobre el nido del cuco) } & Fracaso & $0-7 \%$ & \\
\hline & Éxito notable & $36-75 \%$ & \\
\hline \multirow{2}{*}{ PF3 (La naranja mecánica) } & Fracaso & $0-9 \%$ & $0-9 \%$ \\
\hline & Éxito notable & $61-73 \%$ & $65-100 \%$ \\
\hline
\end{tabular}

Rev. Med. Cine. 2020; 16(4), 297-305 Ediciones Universidad de Salamanca / @@@ 


\section{USO DEL CINE COMERCIAL EN LA ENSEÑANZA DE PSICOLOGÍA PARA ESTUDIANTES DE MEDICINA Y DE BIOLOGÍA JORGE PÉREZ; EVA BAILLÈS}

Tabla 4. Correlaciones entre el resultado de las evaluaciones formativas y de las PEM sumativas finales.

\begin{tabular}{|l|l|l|l|l|}
\hline \multicolumn{1}{|c|}{ Universidad } & \multicolumn{1}{c|}{ Curso académico } & \multicolumn{1}{c|}{ PF1 } & \multicolumn{1}{c|}{ PF2 } & \multicolumn{1}{c|}{ PF3 } \\
\hline \multirow{4}{*}{$\begin{array}{l}\text { UAB } \\
\text { Medicina }\end{array}$} & $1995-96(n=46)$ & $0,38^{* *}$ & $0,42^{* *}$ & $0,48^{* *}$ \\
\cline { 2 - 5 } & $1996-97(n=65)$ & $0,31^{*}$ & $0,30^{*}$ & $0,32^{*}$ \\
\cline { 2 - 5 } & $1997-98(n=30)$ & $0,40^{*}$ & $0,49 * *$ & $0,53^{* *}$ \\
\hline \multirow{4}{*}{$\begin{array}{l}\text { UPF } \\
\text { Biología }\end{array}$} & $2001-02(n=27)$ & 0,04 & - & $0,58^{* *}$ \\
\cline { 2 - 5 } & $2002-03(n=40)$ & $0,36^{*}$ & - & $0,63^{* *}$ \\
\cline { 2 - 5 } & $2003-04(n=42)$ & $0,31^{*}$ & - & $0,52^{* *}$ \\
\cline { 2 - 5 } & $2004-05(n=31)$ & 0,14 & - & $0,43^{*}$ \\
\cline { 2 - 5 } & $2005-06(n=36)$ & 0,22 & $0,54 * *$ \\
\cline { 2 - 5 } & $2006-07(n=27)$ & $0,55^{* *}$ & 0,32 \\
\hline
\end{tabular}

PF1= El milagro de Anna Sullivan; PF2= Alguien voló sobre el nido del cuco; PF3=La naranja mecánica; * p<0,05; $* * p<0,01$

En la tabla 4 se muestran las correlaciones de Pearson entre las calificaciones de las pruebas formativas y las obtenidas en las PEM sumativas finales. Éstas siempre fueron positivas y en numerosos casos significativas. Así mismo, salvo mínimas excepciones, las correlaciones más bajas se daban en la primera prueba y las más altas en la última.

\section{Discusión}

Numerosas experiencias han mostrado la utilidad de usar películas populares como herramienta para favorecer el aprendizaje de materias muy diversas ${ }^{20}$. También sabemos de la utilidad de las evaluaciones formativas que, si bien no tienen contingencia sobre la nota, permiten tanto al alumno como al profesor conocer la situación de aprendizaje durante el proceso docente ${ }^{21}$.

Desde hace bastantes años, nosotros hemos utilizado películas populares como elemento clave para realizar evaluaciones formativas. Sabemos que éstas favorecen el aprendizaje y, por consecuencia, los resultados en las evaluaciones sumativas ${ }^{21,22}$.
De nuestra experiencia hacemos una valoración muy favorable no solo a partir de la satisfacción de los estudiantes, sino de su rendimiento académico. Los resultados en las evaluaciones formativas siempre han sido muy positivos y estamos convencidos que dichas evaluaciones, incluyendo sus sesiones de retroalimentación (feedback), han favorecido los resultados finales de nuestros estudiantes. El éxito encontrado tanto en las evaluaciones formativas como sumativas no nos extraña ya que el tipo de alumnado tanto de Medicina como de Biología ha sido siempre muy brillante y motivado.

Las correlaciones entre los resultados de las evaluaciones formativas y los obtenidos en la prueba final acreditativa PEM, totalmente objetiva, siempre fueron positivas y en la mayoría de los casos significativas. Además, hemos podido observar que las correlaciones son más altas en la última prueba formativa, donde estarían más consolidados los aprendizajes, este hecho avalaría el posible efecto favorable de la evaluación formativa sobre la acreditativa.

Rev. Med. Cine. 2020; 16(4), 297-305 Ediciones Universidad de Salamanca / @®@ J. Med. Mov., 2020; 16(4), 297-305 


\section{USO DEL CINE COMERCIAL EN LA ENSEÑANZA DE PSICOLOGÍA PARA ESTUDIANTES DE MEDICINA Y DE BIOLOGÍA JORGE PÉREZ; EVA BAILLÈS}

Respecto a las películas usadas estamos muy satisfechos ya que, además de ser amenas, recordamos que todas tuvieron un gran éxito comercial en su momento, y presentan relaciones interpersonales que contemplan todos los temas previstos en nuestros objetivos docentes.

Como elemento más relevante de este escrito quisiéramos destacar la posibilidad que tiene el uso de películas populares para realizar evaluaciones de los aprendizajes, bien formativas, como hemos hecho en la actividad presentada, o bien sumativas, como habitualmente lo habíamos hecho en una asignatura introductoria en los estudios de Biología de la UPF ${ }^{23}$.

Finalmente, nuestra experiencia en la enseñanza de Psicología a estudiantes de Medicina y de Biología también avala el uso del cine comercial como herramienta de aprendizaje en la docencia universitaria.

\section{Referencias}

1. Böhnke M, Machura S. Young Tom Edison-Edison, the man: biopic of the dynamic entrepreneur. Public Underst Sci 2003; 12(3): 319-33.

2. Leet D, Houser S. Economics goes to Hollywood: using classic films and documentaries to create an undergraduate economics course. J Econ Edu 2003; 34(4): 326-32.

3. English FW, Steffy BE. Using film to teach leadership in Educational administration. Educ Adm Q 1997; 33(1): 107-15.

4. Davis A. Using feature films in Rehabilitation counsellor education. Rehab Edu 2000; 14(2): 169-80.

5. Landin M. The Name of the Rose / Der Name der Rose (1886). Basic aspects of monastic medical-pharmacological knowledge in de middle ages. J Med Movies [Internet] 2008; 4(2): 41-6.

6. Baños JE, Aramburu J Sentí M. Biocinema: the experience of using popular movies with students of Biology. J Med Movies [Internet] 2005; 1(2): 42-6.
7. Rose, C. (2007). Biology in the Movies: using the double-edged sword of popular culture to enhance public understanding of science. Evol Biol, 34(1): 49-54.

8. Rey Torné U, López Guimet J, Jansà Mas A, Pardo Pastor C, Valmaseda Freixa A, Pérez J. Cine y Biología: Una experiencia docente realizada por los estudiantes. Rev Med Cine [Internet] 2013: 9(2), 82-7.

9. Alexander M, Lenahan P, Pavlov A. Cinemeducation: a comprehensive guide to using film in medical education. Oxford: Radcliffe Publishing; 2005.

10. Kelley M, Calkins S. Evaluating popular portrayals of memory in film. Teach Psychol 2006; 33(3): 191-4.

11. Bhagar HA. Should cinema be used for medical student education in psychiatry? Med Edu 2005; 39(9): 972-73.

12. Flening $\mathrm{MZ}$, Piedmont RL, Hiam CM. Images of madness: feature films in teaching psychology. Teach Psychol 1990; 17(3): 185-7.

13. Paddock JR, Terranova S, Giles L. SASB goes Hollywood: teaching personality theories through movies. Teach Psychol 2001; 28(2): 117-21.

14. Anderson DD. Using films as tool for analysis in a Psychology and Law course. Teach Psychol 1992; 19(3): 155-8.

15. Urra J. Comentario de películas. Anuario Psicol Jur 2003; 13(1): 137-56.

16. Desforges DM. Applying theories of development: an exercise for teaching Adolescent Psychology. Teach Psychol 1994; 21(4): 245-6.

17. Conner DB. From Monty Python to Total Recall: A feature film activity for the Cognitive Psychology Course. Teach Psychol 1996; 23(1): 33-5.

18. Kirsh SJ. Using animated films to teach Social and Personality development. Teach Psychol 1998; 25(1): 49-51.

19. Casper WJ, Champoux JE, Watt JD, Bachiolchi PD, Schleicher DJ, Bordeaux C. Feature film as a resource in teaching I-O Psychology. Industrial-Organitational Psychol 2003; 41(1): 83-95.

Rev. Med. Cine. 2020; 16(4), 297-305 Ediciones Universidad de Salamanca / @@@ J. Med. Mov., 2020; 16(4), 297-305 


\section{USO DEL CINE COMERCIAL EN LA ENSEÑANZA DE PSICOLOGÍA PARA ESTUDIANTES DE MEDICINA Y DE BIOLOGÍA JORGE PÉREZ; EVA BAILLÈS}

20. Pérez J, Aramburu, J, Baños JE, Bosch F, Díez J, Farré $M$, Girvent $M$, Sentí $M$, Valverde $O$. Uso del cine comercial como herramienta docente en estudios en ciencias de la salud. Una experiencia multidisciplinar y colectiva. FEM 2014, 17 (3), 131-5.

21. Rushton A. Formative assessment: A key to deep learning? Med Teach 2005; 27(6): 509-13.
22. Carrillo-de-la-Peña, MT, Baillès E, Caseras X, Martínez $A$, Ortet $G$, Pérez J. Formative assessment and academic achievement in pre-graduate students of health sciences. Adv Health Sci Educ 2009; 14(1): 61-7.

23. Pérez J. El uso de la película Despertares (1990) para evaluar aprendizajes en la licenciatura de Biología de la Universidad Pompeu Fabra. Diez años de experiencia. Rev Med Cine [Internet] 2009; 5(1): 27-9.

\begin{tabular}{|l|l|}
\hline & $\begin{array}{l}\text { Jorge Pérez Sánchez. Profesor jubilado. Fue Profesor Titular de Psicología } \\
\text { Médica y director de la Oficina Educativa de la Facultad de Ciencias de la } \\
\text { Salud y de la Vida de la UPF. Fue decano de dicha facultad entre } 1999 \text { y } \\
2011 \text { y recibió el premio a la trayectoria docente del Consejo Social de la } \\
\text { UPF en 2006. En } 2011 \text { recibió la distinción Vicens Vives de la Generalitat de } \\
\text { Catalunya a la calidad docente. Actualmente es colaborador del Grupo de } \\
\text { Investigación educativa en Ciencias de la Salud (GRECS) de la UPF. }\end{array}$ \\
\hline $\begin{array}{l}\text { Eva Baillès Lázaro es doctora en Psicología por la Universidad Pompeu Fabra. } \\
\text { Actualmente ejerce como psiconcóloga en el Hospital de Nostra Senyora } \\
\text { de Meritxell de Andorra. Previamente había sido profesora asociada en } \\
\text { las Facultades de Medicina y de Psicología de la Universidad Autónoma } \\
\text { de Barcelona, y en la Facultad de Ciencias de la Salud y de la Vida de la } \\
\text { Universidad Pompeu Fabra. }\end{array}$ \\
\hline
\end{tabular}

Rev. Med. Cine. 2020; 16(4), 297-305 Ediciones Universidad de Salamanca / @@@ J. Med. Mov., 2020; 16(4), 297-305 